\title{
Bandwidth Efficient Mobility Management for Heterogeneous Wireless Networks
}

\author{
Karl Andersson, Daniel Granlund, Muslim Elkotob, Christer Åhlund \\ Luleå University of Technology \\ Division of Mobile Networking and Computing \\ SE-931 87 Skellefteå, Sweden \\ \{karl.andersson,daniel.granlund,muslim.elkotob,christer.ahlund\}@ltu.se
}

\begin{abstract}
An important feature of the upcoming fourth generation wireless networks is support for heterogeneous radio access technologies in combination with an all-IP type of overall architecture. Operators and users will benefit from a smooth technology transition leveraging existing investments and use a variety of access technologies simultaneously.

This paper describes and evaluates an innovative mobility management scheme in such an environment. It does not require any changes to the IP stack in the mobile node and does not introduce any additional overhead to the payload traffic over air interfaces. Furthermore, it does not add any signaling overhead and outperforms existing mobility management schemes for heterogeneous environments in terms of bandwidth consumption. The architecture uses a make-before-break principle for vertical handovers and bidirectional tunneling using various tunneling mechanisms connecting mobile nodes through access networks to a home network. Also, it proposes a packet inspection routine for timely handover execution in the home network.
\end{abstract}

The architecture is evaluated both through analytical calculations and experiments using a voice over IP traffic model.

Keywords: Network-based mobility management; Heterogeneous networking environments; VoIP applications

\section{INTRODUCTION}

Future wireless networking environments will be built up of a variety of access technologies with overlapping coverage areas. At the same time, handsets typically already today support two or more access technologies. Furthermore, the upcoming $4 \mathrm{G}$ type of networks will support heterogeneous access and be built up in an all-IP fashion. This way, operators may have the opportunity to perform a smooth transition into new technologies and leverage existing investments. Also, end-users will benefit from having the opportunity to connect to various types of access networks optimizing capacity, cost, or any other parameter of interest.

Within the aforementioned environment a number of issues need to be solved including efficient spectrum allocation, core network design allowing heterogeneous access and all-IP features, as well as a management framework for provisioning of operator policies. Also, a bandwidth efficient mobility management scheme offering seamless mobility to end-users will be needed.

Mobility management was traditionally implemented within the access networks themselves, so that when users roamed over to another access network and traveled across IP subnets, the IP address was typically changed. TCP connections do not survive such changes and connection-less applications need to reinitiate in one way or another. IP mobility management solves this issue by decoupling the IP address from the physical attachment to the Internet.

Mobile IP implements IP mobility management by using two IP addresses where one is referred to as the home address (HoA) which is stable over time and should be regarded as an endpoint identifier. The other IP address is the care of address (CoA) which varies over time while the user is roaming among access networks and reflects the location of the user at any time. The operation of Mobile IP involves mobile nodes (MNs) to regularly register its locations to the home agent (HA) placed in the home network. Registrations are sent in binding update (BU) messages and acknowledged in binding acknowledgements (BAck). The HA is a specialized router tunneling packets for any connected $\mathrm{MN}$ to its current location. The IP stack of the MN is changed so that packets are encapsulated and decapsulated and that registration messages are sent periodically.

Mobile IP is implemented both for IPv4 [1] and IPv6 [2] and has been improved and optimized with fast handovers [3] and hierarchical bindings [4]. Mobile IPv4 (MIPv4) uses foreign agents (FAs) in the access networks and allows the MN either to use a CoA acquired through normal DHCP mechanisms (also referred to as co-located care of addresses) or by using the FA's address. Mobile IPv6 (MIPv6) does not use FAs, but tunnels all traffic directly to and from the MN. It should also be noted that MIPv6 allows for route optimization, which MIPv4 does not. Drawbacks of Mobile IP include introduction of tunneling overhead in the air interface (applies to MIPv4 with co-located care of addresses and to MIPv6), the presence of signaling overhead, and requirements on changes to the IP stack in the MN.

Apart from Mobile IP, there are also a number of mobility protocols on upper layers including Streamed Control Transmission Protocol (SCTP) [5] with enhancements for mobility (mSCTP) [6] working at the transport layer. Despite its very interesting support for multihoming the use of that solution has not reached higher levels due to required changes in existing well-known TCP-based applications due to replaced transport layer.

Furthermore, application layer mobility was proposed by 


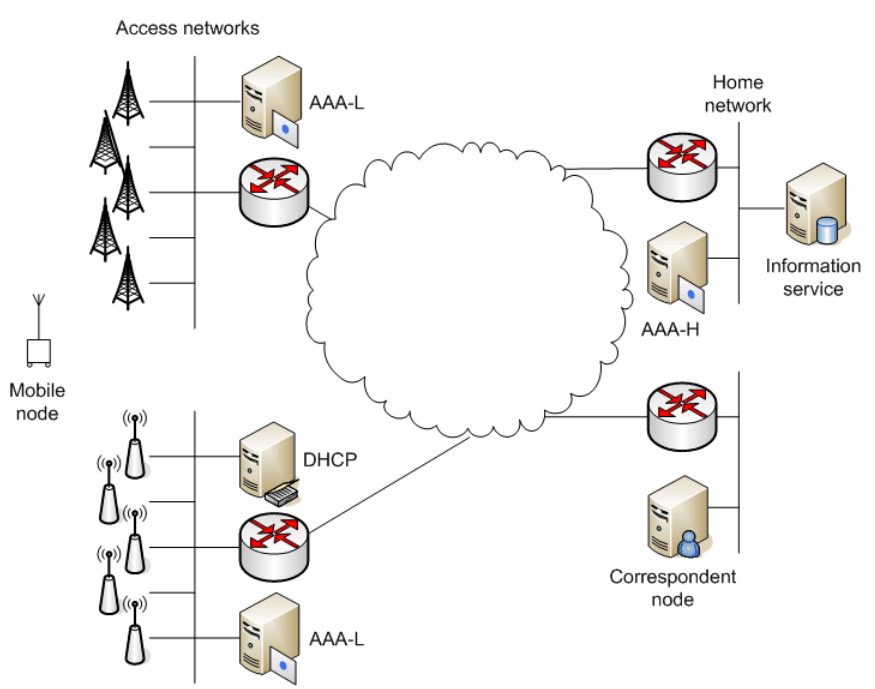

Figure 1. Architecture overview

Schulzrinne et al. [7] adding some features to the Session Initiation Protocol (SIP) [8]. High signaling delay and requirements on implementation of session handling are drawbacks of that solution.

Finally, some standardization bodies are currently active in the area. The Internet Engineering Task Force (IETF) is working on Network-based Localized Mobility Management (NetLMM), where Proxy Mobile IPv6 (PMIPv6) [9] is the concrete solution being proposed. Combining good performance in terms of handover latencies and not requiring any modifications to the mobile node makes PMIPv6 a popular choice.

Keeping those arguments in mind, this paper proposes a network layer mobility management scheme building on previous work $[10,11,12]$. It proposes and evaluates a new efficient scheme having the IP address to stay stable while the user moves across IP subnets, and also avoids tunneling overhead in the wireless parts of the access networks. Furthermore, it does not use any explicit signaling at all. An in-depth comparison between PMIPv6 as well as other existing mobility management solutions and our proposed scheme is provided in Section V.

The rest of the paper is organized in the following way: Section II describes the proposed architecture while Section III describes the evaluation set-up. Section IV covers results while Section V surveys related work. Finally in Section VI the findings will be discussed.

\section{PROPOSED ARCHITECTURE}

\section{A. Overall architecture}

The core contribution of this paper is the architectural proposal presented in this section. The architecture consists of mobile nodes, access networks and one home network (figure 1). Any user is assigned one IP address in the home network and uses only this IP address regardless of where the user chooses to attach to the Internet. An Authentication, Authorization and Accounting (AAA) server in the home network maintains mappings of user names to IP addresses

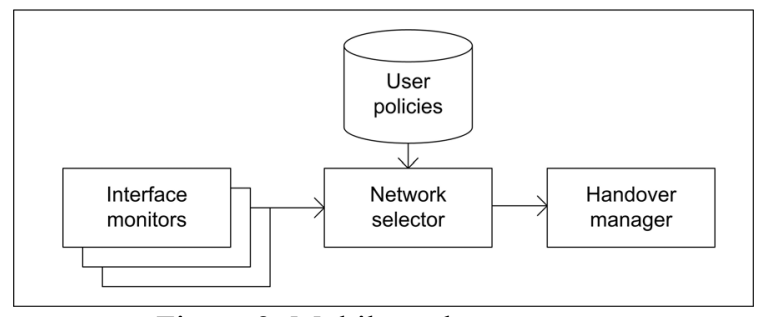

Figure 2. Mobile node components

along with user credentials and other per-user data.

The architecture is mobile node-centric and allows the enduser to perform access network selection based on any principle. When the user attaches to a new access network either PPP-based [13] or IEEE 802.1x-based [14] mechanisms are used to authenticate the user, assign the IP address (which is the same regardless of selected access), and authorize access to the network. The IP address is allocated within the home network and is associated with the user profile. Both mechanisms typically use user@realm type of identification schemes and allow access networks to take various actions when different users log on depending on the user credentials presented to the access network.

Then the access network connects the user to the home network using tunneling techniques such as Layer 2 Tunneling (L2TP) or Generic Routing Encapsulation (GRE), both enabling bidirectional tunneling to the home network. Routing is handled by the access router in the access network and the mobility management entity in the home network. This way, tunneling and signaling overhead are removed from the air interface and there are no requirements on changing the IP stack in the MN. At the same time, it should be noted that a requirement is that all access networks need to implement tunneling capabilities and to set up a connection and trust to the home network.

\section{B. Mobile node details}

Since the MN uses a standard IP stack, there are no mandatory changes needed in the $\mathrm{MN}$ operation or set-up. However, we propose to include interface monitors periodically checking the link quality of each network interface and a network selector (decision engine) to be integrated in the architecture (figure 2). Furthermore, it may be beneficial to implement user policies into the $\mathrm{MN}$ allowing the end-user to set priorities among various parameters like bandwidth, cost, and/or power consumption. Finally, a handover manager being the entity executing handover commands is also part of the architecture.

It should be noted that in this proposal route optimization is not used. This decision was taken to avoid violating the principle of having the IP stacks in nodes unchanged.

\section{Access network details}

The architecture is access technology agnostic and poses no requirements on the access technology itself. However, it must support authentication and authorization implementing AAA$\mathrm{L}$ functionality and to use standard AAA protocols like 


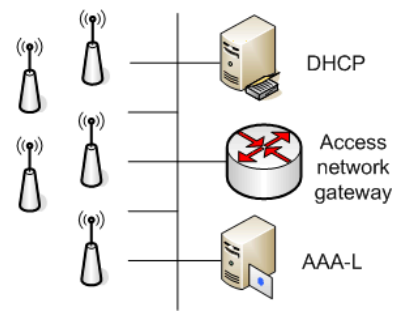

Figure 3. Access network

RADIUS or DIAMETER for communication with the AAA-H entity in the home network (figure 3).

The MN initiates the connection when it senses an active interface. For PPP-based connections LCP and NCP negotiations with authentication e.g. CHAP takes care of this. For 802.11 EAP is used to establish the connection. The same AAA-H is used in both cases. For the case of EAP-based authentication the IP address associated with the user in the home network is delivered by a DHCP server in the access network using information from the MAC layer authentication mechanism performed prior to layer 3 attachment. The AAA mechanism for 802.11-based accesses was described in detail by Granlund et al. [15].

Finally, the access network gateway must support at least one tunneling mechanism such as Layer 2 Tunneling (L2TP) [16] or Generic Routing Encapsulation (GRE) [17] connecting the MN to the home network.

\section{Home network details}

The home network consists of a tunnel factory implementing the central mobility management entity (figure 4). The tunnel factory keeps track of each $\mathrm{MN}$ in the routing table and routes incoming traffic to the appropriate tunnel. When packets arrive from an $\mathrm{MN}$ over a new tunnel, a route command is fired. This way, the tunnel factory follows the access network selection made by the MN continually.

Also, AAA-H functionality is implemented in the home network. Any connection attempt to any access network is centrally handled by the AAA-H entity in the home network authenticating and authorizing users. IP address allocation is also managed by the AAA-H entity, communicating with

AAA-L functionality in the access networks using RADIUS [18] and DIAMETER [19] protocols. Finally, an Information service is hosted in the home network offering MNs to download information on access networks on location of access points, coverage areas, capacity, and other network parameters.

\section{EVALUATION SET-UP}

The proposal was evaluated both through analytical calculations and real-world experiments. The experimental environment was composed of a number of access networks and a home network using tunneling for connecting access networks with the home network (figure 5). The MN and the central mobility management entity were implemented using the Fedora core 10 platform. Equipment from Cisco Systems and their consumer division Linksys were used to create a

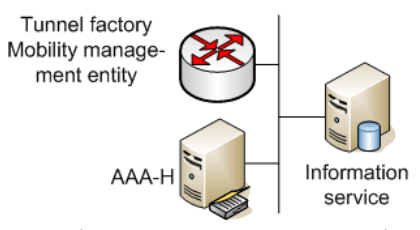

Figure 4. Home network

WLAN access network connected to the home network using GRE tunneling and authenticating and authorizing users using EAP. Also a public CDMA2000 network was used implementing L2TP tunneling to the home network and PPPbased mechanisms (NCP) for authenticating and authorizing end-users.

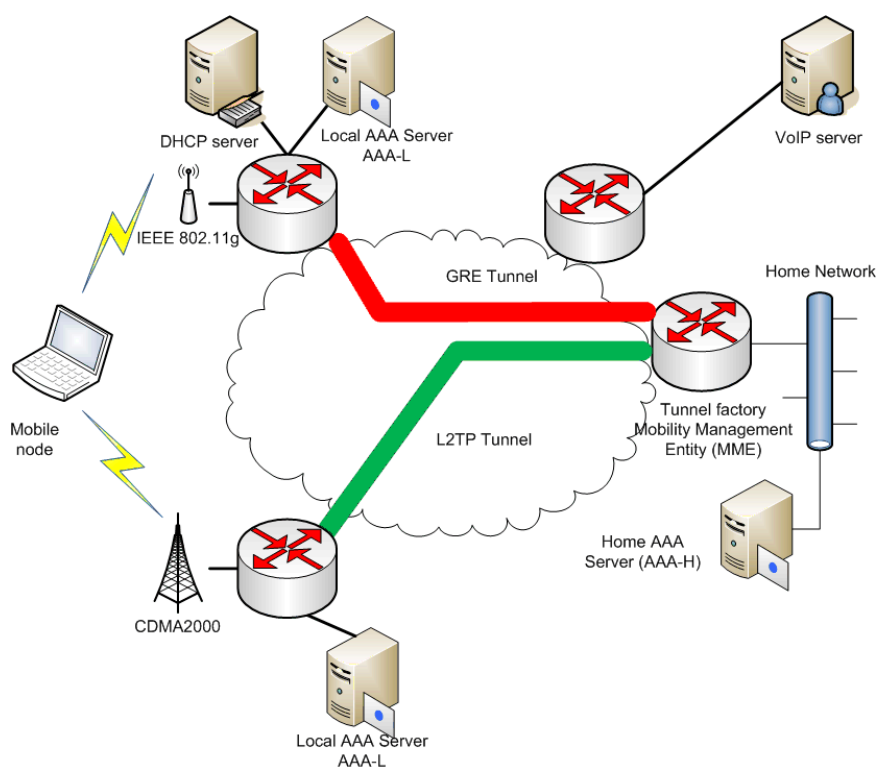

Figure 5. Evaluation set-up

Experiments were performed both in a laboratory testbed where forced handovers were executed repeatedly and in a live environment where an $\mathrm{MN}$ was installed into a car traveling around in an area with overlapping wireless network coverage. Both types of experiments studied a voice over IP (VoIP) application sending traffic to a VoIP server located in the testbed measuring the mean opinion score (MOS) [20] metric. Overall performance was studied at the time of vertical handovers including handover latencies, packet loss rates, and quality degradation of the ongoing VoIP call.

\section{RESULTS}

This section presents analytical calculations of the savings in bandwidth comparing the proposed architecture with a standard Mobile IP architecture (Mobile IPv4 with co-located care of addresses) being the most popular IP mobility management scheme in current research.

\section{A. Analytical calculations of savings in bandwidth}

Since the proposed architecture removes the tunneling overhead from the air interface and also its signaling overhead that is present in Mobile IP there is a significant bandwidth saving potential. Depending on the traffic pattern various 


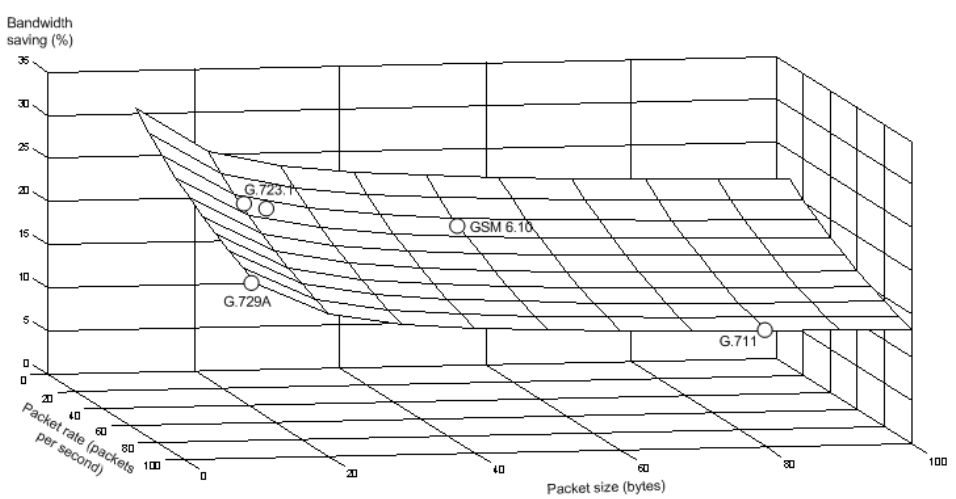

Figure 6. Bandwidth savings in percentage for various codecs

levels of bandwidth saving may be accomplished. We decided to focus on the studied VoIP application since voice traffic is believed to be eventually moved from the PSTN to the Internet. The calculations vary depending on the chosen codec because of differences in packet sizes and packet rates and compare our solution with a standard Mobile IPv4 set up using co-located IP addresses being the most deployed IP mobility scheme at present. Also, we would like to point out that considerable savings are achieved for other Mobile IP configurations, like Mobile IPv6.

In order to analyze the bandwidth saving potential over the air interface, the following equations for bandwidth consumption were identified:

$$
\begin{gathered}
b_{\text {Our scheme }}=r_{\mathrm{Codec}}\left(h_{\mathrm{PHY}}+h_{\mathrm{MAC}}+h_{\mathrm{IP}}+h_{\mathrm{UDP}}+h_{\mathrm{RTP}}+s_{\mathrm{Codec}}\right) \\
b_{\text {MobileIP }}=r_{\mathrm{Codec}}\left(h_{\mathrm{PHY}}+h_{\mathrm{MAC}}+2 h_{\mathrm{IP}}+2 h_{\mathrm{UDP}}+h_{\mathrm{RTP}}+s_{\mathrm{Codec}}\right)+ \\
+r_{\mathrm{MIPsignalling}}\left(s_{\mathrm{BU}}+h_{\mathrm{PHY}}+h_{\mathrm{MAC}}+h_{\mathrm{IP}}+h_{\mathrm{UDP}}\right)
\end{gathered}
$$

where $b$ is bandwidth, $r$ is packet rate for each codec, $h$ is header size for each layer, $s$ is payload packet size for each

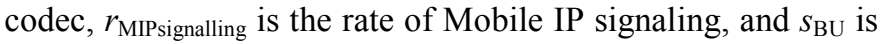
the size of the binding update and acknowledgement messages being present in Mobile IP.

Figure 6 shows the bandwidth saving levels at the physical layer for codecs using various packet sizes and packet rates using (1) and (2). Bandwidth saving rates (in percentage) for commonly used codecs as GSM 6.10, G.711, G.723.1, and G.729A when compared to a standard Mobile IP architecture are found in table 1. The calculations are based on the tunneling and two-way signaling (BU/BAck) overhead for Mobile IP taking an IEEE $802.11 \mathrm{~b} / \mathrm{g}$ type of air interface into account.

\section{B. Results from experiments}

As mentioned in the beginning of this section, the testbed being used contained MNs equipped with CDMA2000 and WLAN access cards, a public CDMA2000 network [21] with EVDO Rev.A capability, a WLAN network with IEEE $802.11 \mathrm{~g}$ capability, and a home network implementing the central mobility management entity and AAA-H functionality. Also, a VoIP server was attached to the testbed in order to
TABLE I

BANDWIDTH SAVINGS FOR VARIOUS VOIP CODECS WHEN COMPARED TO A STANDARD MOBILE IP ARCHITECTURE

\begin{tabular}{|l|r|r|r|r|}
\hline \multirow{3}{*}{ Codec } & \multicolumn{4}{|c|}{ Parameters for each codec and bandwidth saving } \\
\cline { 2 - 5 } & $\begin{array}{c}\text { Source } \\
\text { bitrate } \\
(\boldsymbol{k b} / \mathbf{s})\end{array}$ & $\begin{array}{c}\text { Packet } \\
\text { size } \\
\text { (bytes) }\end{array}$ & $\begin{array}{c}\text { Packet } \\
\text { rate } \\
\text { (pkts/s) }\end{array}$ & $\begin{array}{c}\text { Bandwidth } \\
\text { saving } \\
(\%)\end{array}$ \\
\hline GSM 6.10 & 13 & 32.5 & 50 & $19.9 \%$ \\
\hline G.711 & 64 & 80 & 100 & $14.5 \%$ \\
\hline G.723.1 & 5.3 & 20 & 33.3 & $22.7 \%$ \\
\hline G.723.1 & 6.4 & 24 & 33.3 & $22.1 \%$ \\
\hline G.729A & 8 & 10 & 100 & $21.9 \%$ \\
\hline
\end{tabular}

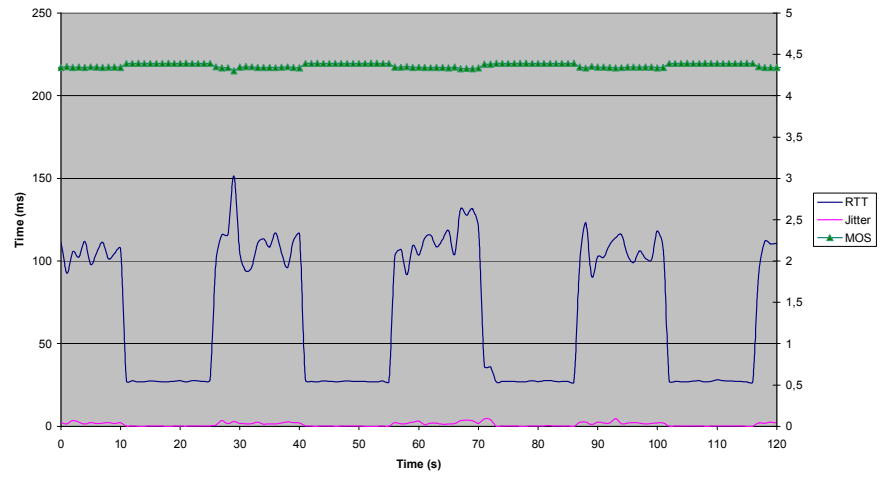

Figure 7. Results from experiments performed in the laboratory

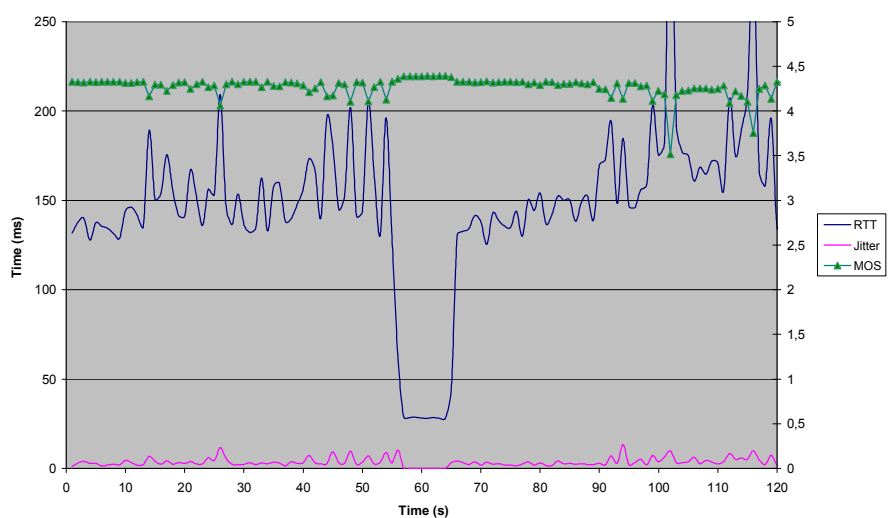

Figure 8. Results from live experiments with $\mathrm{MN}$ in a car

measure MOS values.

In the laboratory experiments conducted, vertical handovers were forced each 15 seconds from CDMA2000 to WLAN and vice versa. Figure 7 shows the results in terms of round trip times (RTTs), jitter, and MOS values achieved in an experiment of 120 seconds duration where a G.711 VoIP application was running on top of the architecture.

The make-before-break type of vertical handover scheme caused packet losses to occur very rarely. Therefore, the MOS value remained high during vertical handovers executed during all experiments. Also, experiments were performed when the MN was installed into a car traveling at around 50 $\mathrm{km} / \mathrm{h}$ in an area with CDMA2000 coverage everywhere and WLAN coverage in smaller areas. The interface monitors and network selector components (figure 2) were of "wlan if 
TABLE II

COMPARISON BETWEEN VARIOUS MOBILITY SOLUTIONS

\begin{tabular}{|l|l|l|l|l|l|l|l|}
\hline \multicolumn{1}{|c|}{ Features } & \multicolumn{1}{|c|}{ MIPv4 } & \multicolumn{1}{|c|}{ MIPv6 } & \multicolumn{1}{|c|}{ SIP } & \multicolumn{1}{c|}{ SCTP } & \multicolumn{1}{c|}{ PMIPv6 } & $\begin{array}{c}\text { Cisco Systems } \\
\text { Proxy Mobile IP }\end{array}$ & \multicolumn{1}{c|}{ Our solution } \\
\hline $\begin{array}{l}\text { Operating } \\
\text { protocol layer }\end{array}$ & Network & Network & Application & Transport & Network & Network \\
\hline $\begin{array}{l}\text { Location } \\
\text { management }\end{array}$ & Yes & Yes & Yes & No & Yes & Yes \\
\hline $\begin{array}{l}\text { Required infra- } \\
\text { structure }\end{array}$ & $\begin{array}{l}\text { Home agent, } \\
\text { Foreign agent }\end{array}$ & Home agent & Registrar & None & $\begin{array}{l}\text { Local Mobility } \\
\text { Anchor, } \\
\text { Mobility } \\
\text { Access } \\
\text { Gateway }\end{array}$ & $\begin{array}{l}\text { Home agent, } \\
\text { Foreign agent, } \\
\text { Access points } \\
\text { supporting } \\
\text { PMIP }\end{array}$ & $\begin{array}{l}\text { Tunnel factory, } \\
\text { Access network } \\
\text { gateway }\end{array}$ \\
\hline $\begin{array}{l}\text { Mobile node } \\
\text { modification }\end{array}$ & Yes & Yes & No & Yes & No & No & No \\
\hline $\begin{array}{l}\text { Handover } \\
\text { latency }\end{array}$ & High & High & High & Low & Yow & No & Now \\
\hline $\begin{array}{l}\text { Route } \\
\text { optimization }\end{array}$ & No & Yes & Yes & No & No & No & No \\
\hline $\begin{array}{l}\text { Tunneling over } \\
\text { wireless link }\end{array}$ & $\begin{array}{l}\text { Scenario } \\
\text { dependent }\end{array}$ & Yes & Now & No \\
\hline
\end{tabular}

coverage" type and implemented so that vertical handovers to WLAN were fired when the signal-to-noise ratio (SNR) for WLAN exceeded a certain threshold. Handovers back to CDMA2000 were fired when another threshold was reached. Using two thresholds in such a way is a feasible way of avoiding ping-pong effects. In addition to the use of two thresholds, a timer was used so that two vertical handovers could not be fired within a certain time frame. Together those two mechanisms make the procedure for vertical handovers stable.

Figure 8 indicates results from one of these experiments where a G.711 VoIP application was running on top of the architecture. A vertical handover from CDMA2000 to WLAN was fired after around 55 seconds and the MN stayed connected to the WLAN access network for about 10 seconds each time.

Also in these experiments the make-before-break vertical handover scheme pays off in terms of low packet losses and stable MOS values. It should be noted that MOS values drop more in the experiments performed in the live environment when compared to the experiments being performed in the controlled laboratory environment.

\section{RELATED WORK}

Gustafsson et al. [22] presented their famous "Always best connected" vision by defining it as a person who is allowed to choose the best available access networks and devices at any time. The authors argued that such environments generate great complexity and a number of requirements, both technical and business oriented. Also, using scenarios they defined a reference model and the actors present including the user, access network operators, service providers, application service providers, and corporations. Access discovery, access selection, AAA support, mobility management, profile handling, and content adaptation were components in the proposal, some of which being implemented in the device and some in the network. Mobility management was based on Mobile IP and SIP in the proposal. No evaluations through simulations or experiments were presented.
Yiping et al. [23] proposed a new architecture for heterogeneous environments including an access discovery mechanism integrating a Service Location Protocol (SLP) and location-based services. Furthermore, it included personalization of network selection through introduction of user weights and constraints in a single objective optimization problem. Also, mobility management was proposed to be based on Mobile IPv6 supporting end-to-end quality of service. Network entities included a Radio Access Directory Agent implementing Directory Agent functionality of the SLP architecture, an ABC agent being the client agent in the SLP architecture, and a network manager collecting QoS information. A guidance network (being a cellular type of wireless network) provided the $\mathrm{MN}$ with location information through a location service server. Operations for seamless handovers were described through an interaction diagram. Evaluations through a proof-of-concept experimental demonstration system were planned but not yet performed in the article, and also AAA handling was left for future work. Our work includes a real world prototype and handles AAA according to what is described in [15].

Nguyen-Vuong et al. [24] proposed a terminal-controlled mobility solution across heterogeneous networks. A network selection mechanism giving users the possibility to select the best access network to maximize their satisfaction was proposed. Also, power-saving interface management was proposed. Mobility management was designed to use either Mobile IPv4 (with FA care of addresses or with co-located care of addresses), or, in an IPv6 environment, to use Mobile IPv6. Interestingly enough, multiple care of addresses were also allowed in the proposal and also, the handover procedure between UMTS and WLAN/WiMAX was clearly described. Interface management and network selection mechanisms were evaluated through simulations. Our paper describes a realworld prototype being used to evaluate the proposed mobility management scheme. Furthermore, it does not require changes to the MN IP stack.

Perera et al. [25] proposed using coexisting mobility protocols in order to support a large range of mobility 
scenarios. By introducing a mobility toolbox enabling mobility management handling to be selected according to the context the authors demonstrated improvements through a prototype based on a scenario implementing network mobility. However, no quantitative results like concrete improvements to handover delays, packet losses, etc. were reported as is the case for our work.

Our proposed solution and PMIPv6 share a set of common design goals, but use different IP protocol versions, with the former being based on IPv4 and the latter on IPv6. Apart from the PMIPv6 standard, Cisco Systems delivers Proxy Mobile IP services as part of its product suite for IEEE 802.11 wireless infrastructure. It is an IPv4-based solution allowing mobile nodes to connect via a foreign agent in the wireless access network to a home agent in the home network. IP addresses are statically assigned to mobile nodes and the solution is only designed for IEEE 802.11. Moreover, the access points need to have special support for subnet mappings.

The proposed solution in this paper delivers network-based mobility management for IPv4 environments with support for a variety of access technologies (such as IEEE 802.11, CDMA2000, UMTS, etc.) and it does not require special support in wireless access points. Furthermore, the solution proposed in this paper enables autoconfiguration of mobile nodes since IP addresses do not need to be configured statically in advance. Table II summarizes different features for some of the mobility management schemes including the one proposed in this paper.

\section{CONCLUSION AND FUTURE WORK}

This paper proposed and evaluated a new mobility management scheme for heterogeneous wireless networks. It was shown that bandwidth savings could reach $30 \%$ at the physical layer for VoIP type of applications when compared to existing standard Mobile IP architectures. Also, this paper showed through experiments that the proposed make-beforebreak type of handover scheme makes user-perceived quality of service for such applications to remain high when vertical handovers take place. Furthermore, we conclude that the simplicity and straightforward implementation details of this proposal make it suitable for fast deployment in real world scenarios.

As future work, we will extend the model, so that locationbased information is fed to the network selector of the MN. Also, we already extended the experimental setup to also include handheld devices like the Apple iPhone. Future work will elaborate such devices even further.

Finally, we intend to extend the proposed architecture with support for Media Independent Handover Services (IEEE 802.21) [26].

\section{ACKNOWLEDGMENT}

The work presented in this paper is based on results from the HybriNet@Skellefteå, Mobile \& Open Service Access, and BasicNet projects supported by Skellefteå Kraft, EU structural funds (Objective 2), and VINNOVA respectively.

\section{REFERENCES}

[1] C. Perkins (ed.), IP Mobility Support for IPv4, IETF, RFC 3344, August 2002

[2] D. Johnson, C. Perkins, and J. Arkko, IP Mobility Support in IPv6, IETF, RFC 3775, June 2004

[3] R. Koodli (ed.), Fast handovers for Mobile IPv6, IETF, RFC 4068, July 2005

[4] H. Soliman, C. Castelluccia, K. El Malki, L. Bellier, Hierarchical Mobile IPv6 Mobility Management (HMIPv6), IETF, RFC, 4140, August 2005

[5] R. Stewart, Q. Xie, K. Morneault, C. Sharp, H. Schwarzbauer, T. Taylor, I. Rytina, M. Kalla, L. Zhang, and V. Paxson, Stream Control Transmission Protocol, IETF, RFC 2960, October 2000

[6] S. J. Koh, M. J. Chang, and M. Lee, mSCTP for Soft Handover in Transport Layer, In IEEE Communications Letters, Volume 8, No. 3, pp. 189-191, March 2004

[7] H. Schulzrinne and E. Wedlund, Application-layer mobility using SIP, Mobile Computing and Communications Review archive, Volume 4, Issue 3, pp. 47-57, July 2000

[8] J. Rosenberg, H.Schulzrinne, G. Camarillo, A. Johnston, J. Peterson, R. Sparks, M. Handley, and E. Schooler, SIP: Session Initiation Protocol, IETF, RFC 3261, June 2002

[9] S. Gundavelli (ed.), Proxy Mobile IPv6, IETF, RFC 5213, August 2008

[10] C. Åhlund, R. Brännström, and A. Zaslavsky, M-MIP: Extended Mobile IP to Maintain Multiple Connections to Overlapping Wireless Access Networks, In Lecture Notes in Computer Science, Volume 3420/2005, pp. 204-213, April 2005

[11] C. Åhlund, R. Brännström, and A. Zaslavsky, Traffic load metrics for multihomed mobile IP and global connectivity, In Telecommunication Systems, Volume 33, Numbers 1-3, pp. 155-185, December 2006

[12] K. Andersson, D. Granlund, and C. Åhlund, M4: MultiMedia Mobility Manager - a seamless mobility management architecture supporting multimedia applications, In ACM International Conference Proceeding Series, Proceedings of the 6th International Conference on Mobile and Ubiquitous Multimedia, MUM2007, Oulu, Finland, December 2007

[13] W. Simpson (ed.), The Point-to-Point Protocol (PPP), IETF, RFC 1661, July 1994

[14] IEEE Standard for Local and metropolitan area networks Port-Based Network Access Control, December 2004

[15] D. Granlund, K. Andersson, M. Elkotob, and C. Åhlund, A uniform AAA handling scheme for heterogeneous networking environments, In Proceedings of the 34th IEEE Conference on Local Computer Networks, LCN 2009, Zürich, Switzerland, October 2009

[16] J. Lau (ed.), M. Townsley (ed.), and I. Goyret (ed.), Layer Two Tunneling Protocol - Version 3 (L2TPv3), IETF, RFC 3931, March 2005

[17] D. Farinacci, T. Li, S. Hanks, D. Meyer, and P. Traina, Generic Routing Encapsulation (GRE), IETF, RFC 2784, March 2000

[18] C. Rigney, S. Willens, A. Rubens, and W. Simpson, Remote Authentication Dial In User Service (RADIUS), IETF, RFC 2865, June 2000

[19] P. Calhoun, J. Loughney, E. Guttman, G. Zorn, and J. Arkko, Diameter Base Protocol, IETF, RFC 3588, September 2003

[20] ITU-T P.880, Telephone transmission quality, telephone installations, local line networks: Methods for objective and subjective assessment of quality, Continuous evaluation of time varying speech quality, May 2004

[21] ice.net

[22] E. Gustafsson and A. Jonsson, Always best connected, IEEE Wireless Communications, Volume 10, Issue 1, pp. 49-55, February 2003

[23] C. Yiping and Y. Yuhang, A new $4 \mathrm{G}$ architecture providing multimode terminals always best connected services, In IEEE Wireless Communications, Volume 14, No. 2, pp. 36-41, April 2007

[24] Q.-T. Nguyen-Vuong, N. Agoulmine, and Y. Ghamri-Doudane, Terminal-Controlled Mobility Management in Heterogeneous Wireless Networks, In IEEE Communications Magazine, Volume 45, No. 4, pp. 122-129, April 2007

[25] E. Perera, R. Boreli, S. Herborn, M. Georgiades, J. Eisl, and E. Hepworth, A mobility toolbox architecture for all-IP networks: an ambient networks approach, In IEEE Wireless Communications, Volume 15, No.2, pp. 8-16, April 2008

[26] IEEE Standard for Local and metropolitan area networks-Part 21: Media Independent Handover Services 